\title{
Über den von Herrn G. Peano aufgestellten Begriff des bestimmten Integrals.
}

\author{
(Nachtrag.) $\left.)^{1}\right)$
}

Von 0. Stolz in Innsbruck.

Hr. Peano stellte seine Definition des bestimmten Integrals bereits 1883 auf. $^{2}$ ) Bald darauf gab auch Hr. $\mathrm{Pasch}^{3}$ ) den derselben zu Grunde liegenden Satz und zwar in Verbindung mit den Formeln

$$
\lim _{\delta_{r}=0} \sum_{r=1}^{r=n} g_{r} \delta_{r}=G, \quad \lim _{\delta_{r}=0} \sum_{r=1}^{r=n} k_{r} \delta_{r}=K,
$$

welche gewöhnlich an die Spitze der Lehre von den bestimmten Integralen gestellt werden.

Wenn nun auch die allgemeinen Eigenschaften des bestimmten Integrals sich ohne Schwierigkeit aus dem Peano'schen Begriffe desselben ergeben, so sind doch die soeben angeführten Formeln keineswegs überflüssig. Man wird nämlich doch wissen wollen, welche Werte man den Theilen $\delta_{1}, \delta_{2}, \ldots, \delta_{n}$ des Integrationsintervalles $b-a$ beizulegen hat, damit der nicht-negative Unterschied

$$
S\left(a, a_{1}, \ldots, a_{n-1}, b\right)-G
$$

unter eine vorgegebene Zahl $\varepsilon>0$ sinkt. Diese Frage kann schwerlich einfacher beantwortet werden, als durch den Satz, dass $S\left(a, a_{1}, \ldots, a_{n-1}, b\right)$ bei unbeschränkter und unbegrenzter Abnahme aller Zahlen $\delta_{1}, \ldots, \delta_{m}$ den Grenzwert $G$ hat.

Ich habe versucht, ob die Peano'sche Definition des einfachen bestimmten Integrals sich nicht auf das eigentliche Doppelintegral auch unter der Bedingung, dass bloß geradlinig-begrenzte Theile des Integrationsgebietes zugelassen werden, ausdehnen lasse; konnte indess nur den folgenden Satz erlangen.

1) Vgl. diese Zeitschrift VII. Jahrg., S. 291.

2) Vgl. Atti della R. Accad. d. scienze di Torino, T. XVIII, S. 441 . Daselbst giebt er auch den Satz I auf S. 293, Bd. VII, den übrigens schon Dini (Teorica $d$. funzioni di var. reali $\mathrm{Nr}$. 185) bemerkt hatte.

3) Vgl. Math. Ann. Bd. 30 (1887) S. 144. Die dort eingeführten Bezeichnungen: "unteres and oberes Integral" wurden bereits von V. Volterra (Battaglini Giornale di Mat. XIX [1881] S. 340) vorgeschlagen. 
„Die für jeden Punkt $x, y$ des endlichen Gebietes $\mathfrak{F}$ eindeutigdefinierte und in diesem Gebiete endliche Function $f(x, y)$ habe in keinem Punkte desselben einen negativen Wert. Über die Fläche $\mathfrak{F}$ werde ein System geradlinig-begrenzter Zellen $\tau_{1}, \ldots, \tau_{n}$ in der Art ausgebreitet, dass sie $\mathfrak{F}$ vollständig bedecken, wobei einige unter ihnen über $\widetilde{F}$ hinausragen dürfen; es sollen also zu jeder der Zellen $\tau_{r}$ Innenpunkte von $\widetilde{F}_{\text {g gehören. Be- }}$ zeichnet man mit $g_{r}$ die obere, mit $k_{r}$, die untere Grenze der Werte von $f(x, y)$ für die Punkte der Zelle $\tau_{r}$ und bildet die Summe

$$
S\left(\tau_{1}, \ldots, \tau_{n}\right)=\sum_{r=1}^{r=n} g_{r} \tau_{r},
$$

so hat dieselbe, wenn man sich den $\tau_{1}, \tau_{\ddot{*}}, \ldots$ alle möglichen Werte beigelegt denkt, eine endliche untere Grenze $G . "$ "

„Bedeutet dann $\tau_{1}^{\prime}, \tau_{2}^{\prime}, \ldots, \tau_{n^{\prime}}^{\prime}$ eine Schar von geradlinigbegrenzten Zellen, welche innerhalb $\mathfrak{F}$ liegen oder doch die Begrenzung von $\mathfrak{F}$ blo $\$$ berühren, $g_{r}^{\prime}$ und $k_{r}^{\prime}$ die obere und untere Grenze von $f(x, y)$ für die Punkte von $\tau_{r}^{\prime}$, und bildet man die Summe

$$
T\left(\tau_{1}^{\prime}, \ldots, \tau_{n^{\prime}}^{\prime}\right)=\sum_{r=1}^{r=n^{\prime}} k_{r}^{\prime} \tau_{r}^{\prime},
$$

so hat sie, wenn man sich den $\tau_{1}^{\prime}, \tau_{2}^{\prime}, \ldots$ alle möglichen Werte beigelegt denkt, eine endliche obere Grenze $K$."

"Es ist dann $\left.G \geq K,{ }^{1}\right)-$ Falls $G=K$ ist, hat die Function $f(x, y)$ ein Doppelintegral über das Gebiet $\mathfrak{F}$ und zwar ist es gleich dem gemeinsamen Werte von $G$ und $K$. $G$ heiBt das obere, $K$ das untere Doppelintegral von $f(x, y)$ über das Gebiet $\mathfrak{F} . "$

Ist im Gebiete $\mathfrak{F}$ dagegen durchaus $f(x, y) \leq 0$, so muss die untere Grenze der Summen

$$
\sum_{u=1}^{r=n^{\prime}} g_{r}^{\prime} \tau_{r}^{\prime}
$$

als oberes, die obere Grenze der Summen

$$
\sum_{r=1}^{r=n} k_{r} \tau_{r}
$$

als unteres Doppelintegral von $f(x, y)$ im Gebiete $\mathfrak{F}$ erklärt werden.

1) Der Nachweis der Beziehung $G \geqq K$ ist auf ähnliche Woise zu führen, wie anf S. 292 d. VII. Jahrg. 